\title{
Prevention of clinically important deteriorations in COPD with umeclidinium/vilanterol
}

\author{
This article was published in the following Dove Press journal: \\ International journal of COPD \\ 24 June 2016 \\ Number of times this article has been viewed
}

\author{
Dave Singh' \\ M Reza Maleki-Yazdi² \\ Lee Tombs ${ }^{3}$ \\ Ahmar lqbal ${ }^{4}$ \\ William A Fahy ${ }^{5}$ \\ Ian Naya ${ }^{5}$
}

'Medicines Evaluation Unit, University of Manchester, University Hospital of South Manchester NHS Foundation Trust, Manchester, UK; ${ }^{2}$ Division of Respiratory Medicine, Women's College Hospital, University of Toronto, ON, Canada; ${ }^{3}$ Precise Approach LTD, London UK; ${ }^{4}$ Respiratory Medical Franchise, GSK, Research Triangle Park, NC, USA; ${ }^{5}$ Respiratory Medicines Development Centre, GSK, Stockley Park, Middlesex, UK

Correspondence: Dave Singh Medicines Evaluation Unit, University of Manchester, University Hospital of South Manchester NHS Foundation Trust, Southmoor Road, Manchester M23 9QZ, UK

Tel +44 I6I 9464052

Fax +44 I6I 946 I459

Email dsingh@meu.org.uk
Background: Minimizing the risk of disease progression and exacerbations is the key goal of COPD management, as these are well-established indicators of poor COPD prognosis. We developed a novel composite end point assessing three important aspects (lung function, health status, and exacerbations) of worsening in COPD. The objective was to determine whether dual bronchodilation with umeclidinium/vilanterol (UMEC/VI) reduces clinically important deteriorations (CIDs) in COPD versus placebo or bronchodilator monotherapy.

Methods: This study is a post hoc analysis of two 24-week trials comparing UMEC/VI 62.5/25 $\mu \mathrm{g}$ with UMEC $62.5 \mu \mathrm{g}$, VI $25 \mu \mathrm{g}$, or placebo (Study A; NCT01313650), or UMEC/VI 62.5/25 $\mathrm{g}$ with tiotropium (TIO) $18 \mu \mathrm{g}$ (Study B; NCT01777334) in patients with symptomatic COPD, without a history of frequent exacerbations. Deterioration was assessed as the time to a first CID, a composite measure defined as a decrease of $\geq 100 \mathrm{~mL}$ in trough forced expiratory volume in 1 second or $\geq 4$-unit increase in St George's Respiratory Questionnaire total score or an on-treatment moderate-to-severe COPD exacerbation.

Results: In Study A, fewer patients experienced a first CID with UMEC/VI (44\%) versus UMEC (50\%), VI (56\%), and placebo (75\%). The risk of a first CID was reduced with UMEC/VI (hazard ratio [HR]: 0.37 [95\% confidence interval, CI: 0.30, 0.45]), UMEC (HR: 0.46 [95\% CI: $0.38,0.56]$ ), and VI (HR: 0.55 [95\% CI: 0.45, 0.66]; all $P<0.001)$ versus placebo, and with UMEC/VI versus UMEC (HR: 0.80 [95\% CI: 0.65, 0.97]; $P<0.05$ ) and versus VI (HR: 0.67 [95\% CI: $0.55,0.81] ; P<0.001)$. In Study B, fewer patients experienced a first CID with UMEC/VI (41\%) versus TIO (59\%). UMEC/VI reduced the risk of a first composite CID by $43 \%$ versus TIO (HR: 0.57 [95\% CI: 0.47, 0.69]; $P<0.001$ ).

Conclusion: This exploratory analysis, using a new assessment of clinical deterioration in COPD, revealed that a majority of symptomatic patients with low exacerbation risk experienced a deterioration during the 24-week study periods. UMEC/VI reduces the risk of a first CID versus placebo or bronchodilator monotherapy.

Keywords: COPD, deterioration, airway stability, UMEC/VI

\section{Introduction}

The management goals of COPD emphasize the need to reduce symptoms, improve health status, increase exercise tolerance, prevent exacerbations, slow disease progression, and reduce mortality. ${ }^{1}$ In patients with moderate-to-severe COPD without a history of frequent exacerbations, bronchodilator monotherapy with long-acting muscarinic antagonist (LAMA) is recommended. ${ }^{1}$ However, most patients receiving long-acting bronchodilator monotherapy still report moderate-to-severe breathlessness and regular rescue medication use. ${ }^{2}$ Consequently, maintenance therapy with fixeddose combinations of LAMA and long-acting- $\beta_{2}$-agonist (LABA) is an alternative treatment option for patients with high symptom burden. ${ }^{3}$ 
The combination of the LAMA, umeclidinium (UMEC), and the LABA, vilanterol (VI), provides significant and clinically relevant improvements in lung function, dyspnea, and health status compared with placebo in patients with COPD. ${ }^{4,5}$ UMEC/VI treatment has also demonstrated greater improvements in lung function and patient-reported outcomes (PROs) compared with the LAMA tiotropium (TIO) ${ }^{4,6}$ Similar improvements in lung function and PROs, and similar safety profiles have been reported for other LAMA/LABA combinations compared with long-acting bronchodilator monotherapy. ${ }^{?}$

Studies comparing bronchodilator combinations with their monotherapy components have not consistently demonstrated statistically significant improvements in PROs. ${ }^{8-10}$ Furthermore, the magnitude of PRO changes has been below the accepted threshold for the minimal clinically important difference (MCID). ${ }^{11}$ However, MCID values have traditionally been used for comparisons with placebo, and as such, the magnitude of improvement possible when comparing active treatments is likely to be smaller than the MCID.

Clinical trials of bronchodilators in COPD normally focus on using MCIDs to determine improvements in lung function and PROs. However, it is also important to understand whether these treatments improve airway stability and prevent disease worsening, such as exacerbations. Exacerbations and rapid deteriorations in lung function are well-established indicators of poor long-term COPD prognosis. ${ }^{12-17}$ Furthermore, deteriorations in health status, measured by a St George's Respiratory Questionnaire (SGRQ) score greater than the $\mathrm{MCID}^{11}$ over 1 year, increase the prospective risk of hospitalizations and mortality during the 2 subsequent years. ${ }^{18}$ Therefore, we have developed an exploratory composite end point (clinically important deterioration [CID]) to assess individual deteriorations in lung function and health status (defined by the accepted MCIDs) as well as the incidence of moderate-to-severe COPD exacerbations. ${ }^{11,19,20}$

Using data obtained from the two largest randomized, controlled, 6-month trials comparing UMEC/VI with placebo, the UMEC/VI bronchodilator monotherapy components (UMEC and VI), or TIO in symptomatic patients with moderate-to-very severe COPD, this post hoc analysis assessed whether a fixed-dose combination of UMEC/VI prolonged the time patients remained free of a CID compared with placebo or long-acting bronchodilator monotherapies.

\section{Materials and methods Study design}

This study used data from two independent retrospective, post hoc analyses of two 24-week, Phase III, multicenter, randomized, double-blind, parallel-group studies. In Study A (DB2113373; NCT01313650), patients were randomized (3:3:3:2) to UMEC/VI 62.5/25 $\mu \mathrm{g}$ (delivering 55/22 $\mu \mathrm{g}$; GSK, Brentford, UK), UMEC $62.5 \mu \mathrm{g}$ (delivering $55 \mu \mathrm{g}$ ), VI $25 \mu \mathrm{g}$ (delivering $22 \mu \mathrm{g}$ ), or placebo, all delivered via the ELLIPTA ${ }^{\mathrm{TM}}$ dry powder inhaler (GSK). ${ }^{4}$ In Study B (ZEP117115; NCT01777334), ${ }^{6}$ patients were randomized (1:1) to receive once-daily UMEC/VI 62.5/25 $\mu$ g inhaled via the ELLIPTA ${ }^{\mathrm{TM}}$ dry powder inhaler or TIO $18 \mu \mathrm{g}$ inhaled via the HandiHaler ${ }^{\circledR}$ (Boehringer Ingelheim, Ingelheim, Germany). ${ }^{6}$ Detailed methodologies for both studies have been previously published. ${ }^{4,6}$ Both studies included in this post hoc analysis were approved by the Chesapeake Institutional Review Board (Columbia, Maryland, USA), as well as each relevant national, regional, or independent ethics committee or institutional review board and conducted in accordance with the Declaration of Helsinki and Good Clinical Practice guidelines.

Key inclusion criteria for both studies included age $\geq 40$ years with a diagnosis of COPD according to the American Thoracic Society/European Respiratory Society guidelines, ${ }^{21}$ a forced expiratory volume in 1 second $\left(\mathrm{FEV}_{1}\right) /$ forced vital capacity ratio of $<0.70$ and a post-salbutamol $\mathrm{FEV}_{1} \leq 70 \%$ of predicted normal, and a modified Medical Research Council Dyspnea Scale score $\geq 2$. Key exclusion criteria included a diagnosis of asthma or other respiratory conditions, hospitalization for COPD or pneumonia within the 12 weeks before visit 1 , and the use of prohibited medications. The primary study end point in both studies was trough $\mathrm{FEV}_{1}$ on day 169 . SGRQ score on day 168 was also determined.

\section{Methods}

The primary end point of this exploratory analysis was the time to a first CID, a composite measure defined as 1) a decrease of $\geq 100 \mathrm{~mL}$ from baseline in trough $\mathrm{FEV}_{1},{ }^{19,20}$ 2) a deterioration in health-related quality of life defined as $\geq 4$-unit increase from baseline in SGRQ total score, ${ }^{11}$ or 3) the occurrence of an on-treatment moderate-to-severe COPD exacerbation (defined as an acute worsening of COPD symptoms requiring the use of additional treatment including oral corticosteroids, antibiotics, emergency department treatment, or hospitalization). Deteriorations in trough $\mathrm{FEV}_{1}$ from baseline were determined using data from seven trial visits post-randomization (days 2, 28, 56, 84, 112, 168, and 169) and SGRQ total score from three study visits (days 28,84 , and 168). Time to first deterioration in each of the components of the composite CID was also assessed. Time to a first sustained composite CID was also determined and defined as a CID occurring on two or more consecutive visits 4 weeks apart 
or for $\geq 50 \%$ of all available subsequent visits. For sustained trough $\mathrm{FEV}_{1}$ decreases, the time between days 168 and 169 was not considered a separate visit; a decrease of $\geq 100 \mathrm{~mL}$ in trough $\mathrm{FEV}_{1}$ before day 168 was considered a sustained decrease if it was maintained at either day 168 or day 169. As protocols from both studies required patients be withdrawn from the study following a moderate-to-severe COPD exacerbation, the first incidence of an exacerbation was documented as a sustained CID. Safety assessment in both studies included the monitoring of adverse events (AEs).

\section{Statistical analyses}

Analyses were performed on the intent-to-treat population, defined as all patients randomized to treatment and who received one or more doses of study medication. A sensitivity analysis was performed on data from Study B, excluding trough $\mathrm{FEV}_{1}$ data at days 2, 56, 112, and 169. Subgroup analyses were performed by COPD severity (Global Initiative for Chronic Obstructive Lung Disease [GOLD] groups B and D) and inhaled corticosteroid (ICS) use at screening (yes/no). All available data were included in the analyses, and no imputations for missing data were performed.

Statistical comparisons were completed for UMEC/VI versus UMEC, VI, or placebo, UMEC or VI versus placebo, and UMEC/VI versus TIO. Hazard ratios (HRs) and confidence intervals (CIs) were derived using a Cox proportional hazards model with covariates of treatment, smoking status at screening, and geographical region.

\section{Results \\ Study populations}

The intent-to-treat populations included 1,532 (Study A) and 905 patients (Study B). Patient demographics and baseline characteristics were similar across treatment groups (Table 1).

\section{Efficacy}

\section{Change in trough FEV and SGRQ total score}

Significant improvements in trough $\mathrm{FEV}_{1}$ and SGRQ total score at the final clinical visit for Studies A and B were demonstrated with UMEC/VI, UMEC and VI versus placebo in Study A, and UMEC/VI versus TIO in Study B; results are shown in Tables S1 and S2, respectively.

\section{Time to first composite CID in Study A}

The incidence of a first composite CID ranged from $44 \%$ (UMEC/VI) to $75 \%$ (placebo) (Table 2; Figure 1A). Compared with placebo, the risk of a first composite CID was statistically significantly reduced by $63 \%$ with UMEC/VI (HR: 0.37 [95\% CI: 0.30, 0.45]), 54\% with UMEC (HR: 0.46

Table I Summary of patient demographics and baseline characteristics (ITT population)

\begin{tabular}{|c|c|c|c|c|c|c|}
\hline \multirow[t]{2}{*}{ Category } & \multicolumn{4}{|l|}{ Study A } & \multicolumn{2}{|l|}{ Study B } \\
\hline & $\begin{array}{l}\text { UMEC/VI } \\
(n=4 \mid 3)\end{array}$ & $\begin{array}{l}\text { UMEC } \\
(n=418)\end{array}$ & $\begin{array}{l}\text { VI } \\
(n=42 I)\end{array}$ & $\begin{array}{l}\text { Placebo } \\
(n=280)\end{array}$ & $\begin{array}{l}\text { UMEC/VI } \\
(n=454)\end{array}$ & $\begin{array}{l}\text { TIO } \\
(n=45 I)\end{array}$ \\
\hline Age (years) & $63.1(8.7)$ & $64.0(9.2)$ & $62.7(8.5)$ & $62.2(9.0)$ & $61.9(8.4)$ & $62.7(8.5)$ \\
\hline Male, n (\%) & $305(74)$ & $298(7 I)$ & $285(68)$ & $195(70)$ & $310(68)$ & $303(67)$ \\
\hline Current smoker at screening, $\mathrm{n}(\%)^{\mathrm{a}}$ & $203(49)$ & $207(50)$ & $199(47)$ & $150(54)$ & $270(59)$ & $243(54)$ \\
\hline Smoking pack-years ${ }^{\mathrm{b}}$ & $46.5(25.8)$ & $46.8(27.0)$ & $44.7(23.2)$ & $47.2(27.2)$ & $44.1(24.4)$ & $44.4(25.0)$ \\
\hline Post-salbutamol \% predicted FEV, & $47.8(13.2)$ & $46.8(13.4)$ & $48.2(13.3)$ & $46.7(12.7)$ & $46.2(13.0)$ & $46.5(12.8)$ \\
\hline Reversible to salbutamol, $\mathrm{n}(\%)^{c}$ & $129(31)$ & $121(29)$ & $155(37)$ & $91(33)$ & $124(27)$ & $142(3 \mid)$ \\
\hline Reversibility to salbutamol (L) & $0.151(0.169)$ & $0.137(0.147)$ & $0.164(0.166)$ & $0.159(0.166)$ & $0.148(0.150)$ & $0.152(0.155)$ \\
\hline \multicolumn{7}{|l|}{ GOLD stage, $\mathrm{n}(\%)$} \\
\hline II & $201(49)$ & $191(46)$ & $197(47)$ & $119(43)$ & $185(4 \mid)$ & $190(42)$ \\
\hline III & $166(40)$ & $172(4 \mid)$ & $179(43)$ & $133(48)$ & $207(46)$ & $206(46)$ \\
\hline IV & $45(\mathrm{II})$ & $54(13)$ & $44(10)$ & $28(10)$ & $62(14)$ & $55(12)$ \\
\hline \multicolumn{7}{|l|}{ GOLD group, $\mathrm{n}(\%)^{\mathrm{d}}$} \\
\hline GOLD B & $184(45)$ & $163(39)$ & $160(38)$ & $106(38)$ & $178(39)$ & $175(39)$ \\
\hline GOLD D & $228(55)$ & $254(61)$ & $260(62)$ & $174(62)$ & $276(6 I)$ & $276(6 I)$ \\
\hline Concurrent ICS use, n (\%) ${ }^{\mathrm{e}}$ & $212(5 I)$ & $219(52)$ & $212(50)$ & $137(49)$ & $247(54)$ & $237(53)$ \\
\hline SGRQ total score at baseline & $48.58(18.24)$ & $48.84(18.23)$ & $49.26(17.84)$ & $51.28(18.12)$ & $49.0(17.1)$ & $48.6(16.8)$ \\
\hline \multicolumn{7}{|l|}{ Exacerbation history, n (\%) ${ }^{\mathrm{f}}$} \\
\hline Moderate exacerbation ${ }^{g}$ & $99(24)$ & $120(29)$ & $125(30)$ & $78(28)$ & $70(15)$ & $80(18)$ \\
\hline Severe exacerbation ${ }^{h}$ & $39(9)$ & $51(12)$ & $53(13)$ & $31(11)$ & $34(7)$ & $28(6)$ \\
\hline
\end{tabular}

Notes: Values are reported as mean (SD) unless otherwise stated. ${ }^{a}$ Reclassified: subject reclassified as current smoker if smoked within 6 months. ${ }^{b}$ Smoking pack-years $=$ (number of cigarettes smoked per day/20) $\times$ number of years smoked. 'Reversibility was defined as an increase in FEV, of $\geq 12 \%$ and $\geq 200 \mathrm{~mL}$ following administration of salbutamol. ' $\mathrm{GOLD}$ group data were not available for one patient in each of the UMEC/VI, UMEC, and VI groups. eICS use was defined as those subjects who were currently taking ICS medications at the screening visit. 'During the 12 months prior to screening. ${ }^{8}$ Exacerbation requiring corticosteroid and/or antibiotic without hospitalization. hExacerbation requiring hospitalization.

Abbreviations: FEV , forced expiratory volume in I second; GOLD, Global Initiative for Chronic Obstructive Lung Disease; ICS, inhaled corticosteroid; ITT, intent-to-treat; SD, standard deviation; SGRQ, St George's Respiratory Questionnaire; TIO, tiotropium; UMEC, umeclidinium; VI, vilanterol. 
[95\% CI: $0.38,0.56]$ ), and $45 \%$ with VI (HR: 0.55 [95\% CI: $0.45,0.66$; all $P<0.001$ ) (Table 2; Figure 2A). Significant reductions in risk with UMEC/VI versus placebo and UMEC versus placebo were observed for clinical deteriorations of all three individual parameters $(P<0.05)$ (Table 2). The risk of clinical deteriorations in trough $\mathrm{FEV}_{1}$ and SGRQ total score was statistically significantly decreased with VI versus placebo $(P<0.01)$ (Table 2).

The risk of a first composite CID was statistically significantly reduced with UMEC/VI by $20 \%$ versus UMEC (HR: 0.80 [95\% CI: $0.65,0.97] ; P<0.05$ ) and by $33 \%$ versus VI (HR: 0.67 [95\% CI: 0.55, 0.81]; $P<0.001$ ) (Table 2). The risk of deteriorations in trough $\mathrm{FEV}_{1}$ was statistically significantly reduced with UMEC/VI versus UMEC and VI (Table 2).

\section{Time to first composite CID in Study B}

The incidence of a first composite CID was $41 \%$ with UMEC/VI treatment and 59\% with TIO (Table 3; Figure 1B). UMEC/VI produced a statistically significant reduction of $43 \%$ in the risk of a first composite CID versus TIO (HR: 0.57
[95\% CI: 0.47, 0.69]; $P<0.001$ ) (Table 3; Figure 2B). Statistically significant reductions in risk versus TIO were observed for first deteriorations in all three individual parameters $(P<0.05)$ (Table 3).

\section{Time to sustained composite CID in Study A}

The incidence of a sustained composite CID was approximately half that of a first CID with UMEC/VI (24\%), UMEC (28\%), VI (34\%), and placebo (49\%) (Table 2; Figure 1A). Compared with placebo, there was a statistically significant reduction of $61 \%$ in the risk of a sustained composite CID with UMEC/VI treatment (HR: 0.39 [95\% CI: 0.30, 0.50]), 53\% with UMEC (HR: 0.47 [95\% CI: $0.37,0.60]$ ), and $40 \%$ with VI (HR: 0.60 [95\% CI: 0.47, 0.76]; all $P<0.001$ ) (Table 2; Figure 2C). Statistically significant reductions in the risk versus placebo of a deterioration in trough $\mathrm{FEV}_{1}$ were observed with UMEC/VI, UMEC, and VI $(P<0.001)$ (Table 2). UMEC/VI also produced a statistically significant reduction of $35 \%$ in the risk of a sustained composite CID versus VI (HR: 0.65 [95\% CI: 0.50, 0.84]; $P<0.001$ ) but not with UMEC (Table 2).

Table 2 Summary and analysis of first and sustained CID in study A

\begin{tabular}{|c|c|c|c|c|}
\hline Deterioration criteria & $\begin{array}{l}\text { UMEC/VI } \\
(n=4 \mid 3)\end{array}$ & $\begin{array}{l}\text { UMEC } \\
(n=4 \mid 8)\end{array}$ & $\begin{array}{l}\text { VI } \\
(n=42 I)\end{array}$ & $\begin{array}{l}\text { Placebo } \\
(n=280)\end{array}$ \\
\hline \multicolumn{5}{|l|}{ First deterioration } \\
\hline Composite (any event), n (\%) & $182(44)$ & $210(50)$ & $236(56)$ & $209(75)$ \\
\hline Vs placebo, HR (95\% Cl) & $0.37(0.30,0.45)^{* * *}$ & $0.46(0.38,0.56)^{* * * *}$ & $0.55(0.45,0.66)^{* * *}$ & - \\
\hline UMEC/VI vs UMEC or VI, HR $(95 \% \mathrm{Cl})$ & - & $0.80(0.65,0.97)^{*}$ & $0.67(0.55,0.8 \mathrm{I})^{* * *}$ & - \\
\hline$\geq 100 \mathrm{~mL}$ decrease in trough $\mathrm{FEV}_{\mathrm{l}}, \mathrm{n}(\%)$ & $81(20)$ & $114(27)$ & $145(34)$ & $147(53)$ \\
\hline Vs placebo, HR (95\% Cl) & $0.26(0.20,0.34)^{* * *}$ & $0.40(0.3 \mathrm{I}, 0.5 \mathrm{I})^{* * *}$ & $0.52(0.42,0.66)^{* * *}$ & - \\
\hline UMEC/VI vs UMEC or VI, $\mathrm{HR}(95 \% \mathrm{Cl})$ & - & $0.65(0.49,0.87)^{* *}$ & $0.49(0.38,0.65)^{* * *}$ & - \\
\hline$\geq 4$-unit SGRQ total score increase, $n$ (\%) & $112(27)$ & $120(29)$ & $122(29)$ & $110(39)$ \\
\hline Vs placebo, HR $(95 \% \mathrm{Cl})$ & $0.58(0.44,0.75)^{* * *}$ & $0.63(0.49,0.82)^{* * * *}$ & $0.66(0.5 \mathrm{I}, 0.86)^{* *}$ & - \\
\hline UMEC/VI vs UMEC or VI, HR $(95 \% \mathrm{Cl})$ & - & $0.91(0.71,1.18)$ & $0.87(0.67,1.13)$ & - \\
\hline Moderate-to-severe COPD exacerbation ${ }^{\mathrm{a}}, \mathrm{n}(\%)$ & $27(7)$ & $33(8)$ & $39(9)$ & $35(13)$ \\
\hline Vs placebo, HR (95\% Cl) & $0.48(0.29,0.79) * *$ & $0.60(0.37,0.96)^{*}$ & $0.71(0.45,1.12)$ & - \\
\hline UMEC/VI vs UMEC or VI, HR $(95 \% \mathrm{Cl})$ & - & $0.80(0.48,1.33)$ & $0.68(0.42, \mathrm{I} . \mathrm{II})$ & - \\
\hline \multicolumn{5}{|l|}{ Sustained deterioration } \\
\hline Composite (any event), n (\%) & $100(24)$ & $118(28)$ & $143(34)$ & $137(49)$ \\
\hline Vs placebo, HR $(95 \% \mathrm{Cl})$ & $0.39(0.30,0.50)^{* * *}$ & $0.47(0.37,0.60)^{* * *}$ & $0.60(0.47,0.76)^{* * *}$ & - \\
\hline UMEC/VI vs UMEC or VI, HR $(95 \% \mathrm{Cl})$ & - & $0.83(0.63,1.08)$ & $0.65(0.50,0.84)^{* * *}$ & - \\
\hline$\geq 100 \mathrm{~mL}$ decrease in trough $\mathrm{FEV}_{\mathrm{l}}, \mathrm{n}(\%)$ & $37(9)$ & $49(12)$ & $68(16)$ & $83(30)$ \\
\hline Vs placebo, HR $(95 \% \mathrm{Cl})$ & $0.26(0.17,0.38)^{* * *}$ & $0.34(0.24,0.49)^{* * * *}$ & $0.50(0.36,0.69)^{* * *}$ & - \\
\hline UMEC/VI vs UMEC or VI, HR $(95 \% \mathrm{Cl})$ & - & $0.74(0.49,1.14)$ & $0.5 \mathrm{I}(0.34,0.76)^{* *}$ & - \\
\hline$\geq 4$-unit SGRQ total score increase, $n$ (\%) & $44(\mathrm{II})$ & $49(12)$ & $52(12)$ & $42(15)$ \\
\hline Vs placebo, HR $(95 \% \mathrm{Cl})$ & $0.66(0.43,1.01)$ & $0.74(0.49,1.11)$ & $0.79(0.52,1.18)$ & - \\
\hline UMEC/VI vs UMEC or VI, HR $(95 \% \mathrm{Cl})$ & - & $0.90(0.60,1.35)$ & $0.84(0.56,1.25)$ & - \\
\hline
\end{tabular}

Notes: $* P<0.05$. $* * P<0.01$. $* * * P<0.001$. a Defined as an acute worsening of COPD symptoms requiring the use of additional treatment including oral corticosteroids, antibiotics, emergency department treatment, or hospitalization. ${ }^{b}$ Any first exacerbation was also considered a sustained deterioration as patients were withdrawn by protocol following a first event.

Abbreviations: $\mathrm{Cl}$, confidence interval; CID, clinically important deterioration; FEV , forced expiratory volume in I second; HR, hazard ratio; ITT, intent-to-treat; SGRQ, St George's Respiratory Questionnaire; UMEC, umeclidinium; VI, vilanterol. 

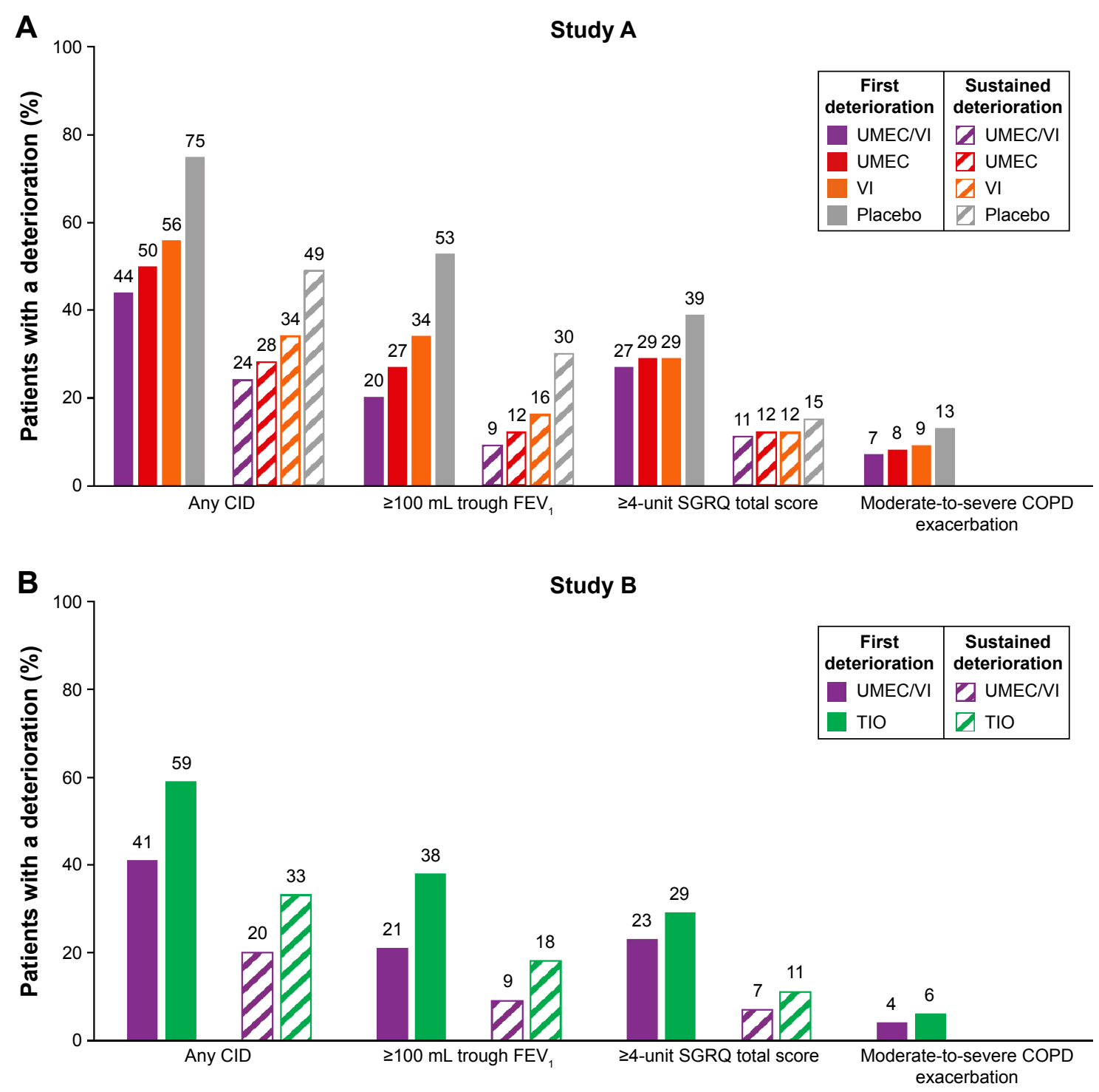

Figure I Proportion of patients with a first and sustained CID (ITT population).

Notes: A moderate-to-severe exacerbation is defined as an acute worsening of COPD symptoms requiring the use of additional treatment including oral corticosteroids, antibiotics, emergency department treatment, or hospitalization.

Abbreviations: CID, clinically important deterioration; FEV , forced expiratory volume in I second; ITT, intent-to-treat; SGRQ, St George's Respiratory Questionnaire; TIO, tiotropium; UMEC, umeclidinium; VI, vilanterol.

\section{Time to sustained composite CID in Study B}

The incidence of sustained composite CIDs was $20 \%$ with UMEC/VI treatment and 33\% with TIO (Table 3; Figure 1B). These values were approximately half that observed for a first composite deterioration (Table 3). UMEC/VI produced a statistically significant reduction of $47 \%$ in the risk of a sustained composite CID (HR: 0.53 [95\% CI: 0.40, 0.68]; $P<0.001$ ) versus TIO (Table 3; Figure 2D). Statistically significant reductions in risk versus $\mathrm{TIO}$ were also observed for sustained deteriorations in trough $\mathrm{FEV}_{1}$ and SGRQ total score $(P<0.05)$ (Table 3$)$.
GOLD severity and concurrent ICS use subgroups in Studies A and B

In Study A, the incidence of a first CID was consistently highest in patients treated with placebo in all subgroups (69\%-80\%) compared with UMEC/VI (40\%-48\%), UMEC (47\%-53\%), and VI (54\%-58\%) (Table S3). All bronchodilator treatments statistically significantly reduced the risk of a first deterioration versus placebo in all subgroups $(P<0.001)$. A statistically significant reduction in the risk of a first composite CID with UMEC/VI versus UMEC and VI in GOLD D and concurrent ICS use subgroups was 


\section{First CID}
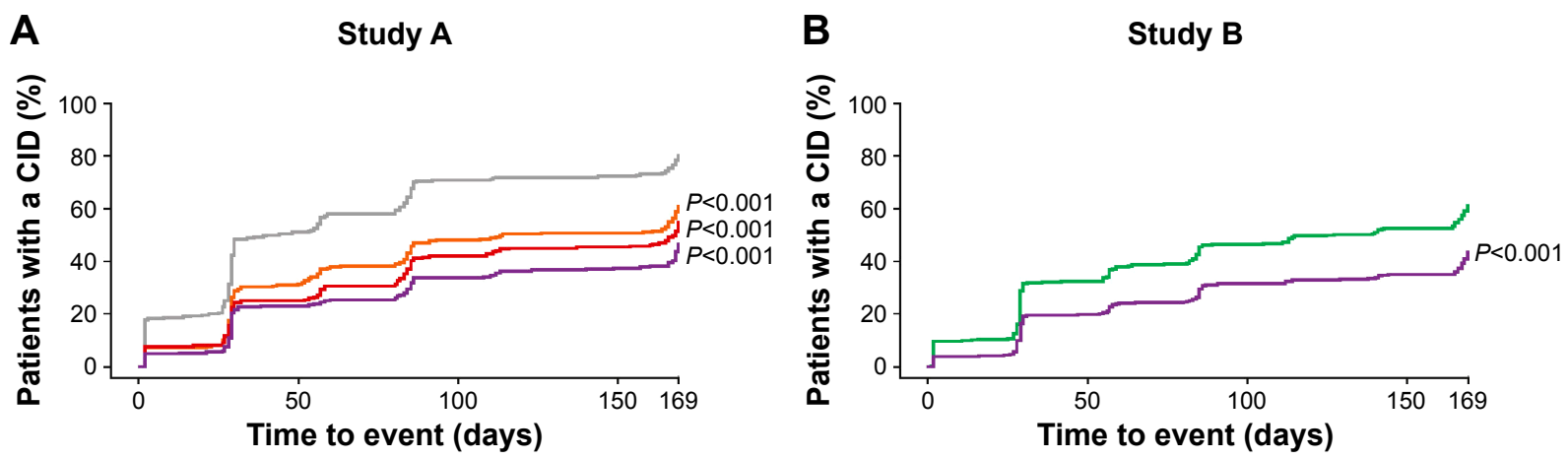

\section{Sustained CID}
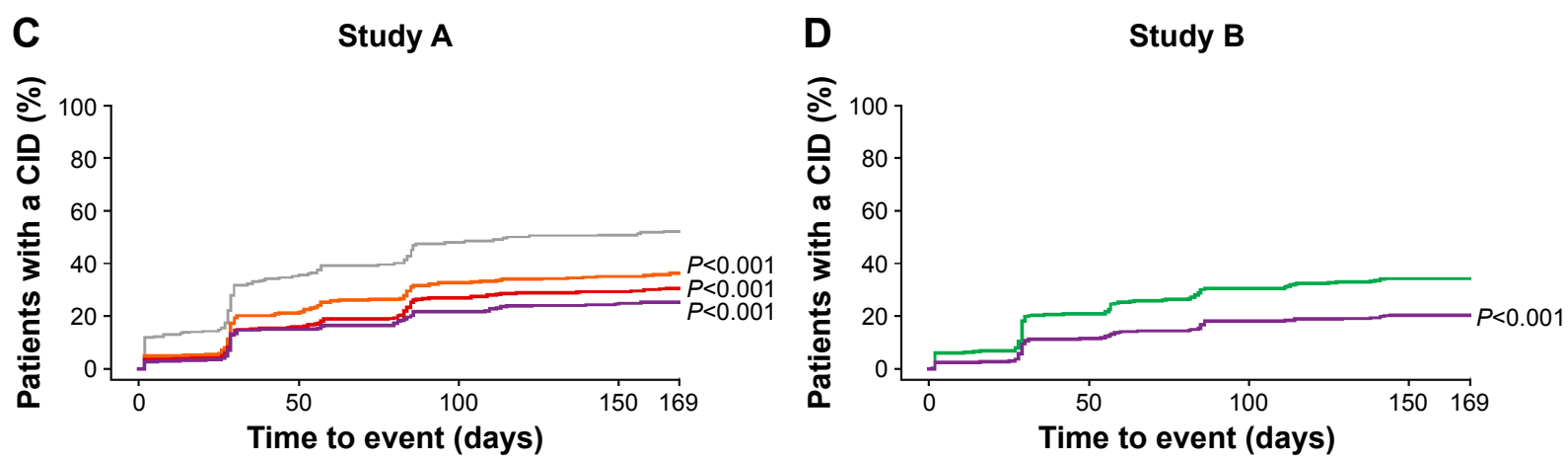

$$
\text { - UMEC/VI - UMEC - VI - Placebo }
$$

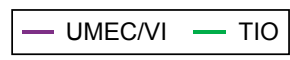

Figure 2 Time to first and sustained CID (ITT population).

Notes: P-values represent comparisons between placebo and UMEC/VI, or UMEC or VI (Study A), or UMEC/VI and TIO (Study B).

Abbreviations: CID, clinically important deterioration; ITT, intent-to-treat; TIO, tiotropium; UMEC, umeclidinium; VI, vilanterol.

Table 3 Summary and analysis of first and sustained CID in Study B (ITT population)

\begin{tabular}{|c|c|c|}
\hline Deterioration criteria & UMEC/VI $(n=454)$ & TIO $(n=45 I)$ \\
\hline \multicolumn{3}{|l|}{ First deterioration } \\
\hline Composite (any event), n (\%) & $188(4 \mid)$ & $266(59)$ \\
\hline UMEC/VI vs TIO, HR (95\% Cl) & - & $0.57(0.47,0.69)^{* * *}$ \\
\hline$\geq 100 \mathrm{~mL}$ decrease in trough $\mathrm{FEV}_{1}, \mathrm{n}(\%)$ & $95(2 \mathrm{I})$ & $170(38)$ \\
\hline UMEC/VI vs TIO, HR (95\% Cl) & - & $0.47(0.36,0.60)^{* * *}$ \\
\hline$\geq 4$-unit SGRQ total score increase, $n$ (\%) & $105(23)$ & $|3|(29)$ \\
\hline UMEC/VI vs TIO, HR (95\% Cl) & - & $0.74(0.58,0.96)^{*}$ \\
\hline Moderate-to-severe COPD exacerbation ${ }^{\mathrm{a}}, \mathrm{n}(\%)$ & $16(4)$ & $29(6)$ \\
\hline UMEC/VI vs TIO, HR $(95 \% \mathrm{Cl})$ & - & $0.53(0.29,0.98)^{*}$ \\
\hline \multicolumn{3}{|l|}{ Sustained deterioration } \\
\hline Composite (any event), n (\%) ${ }^{\mathrm{b}}$ & $90(20)$ & $150(33)$ \\
\hline UMEC/VI vs TIO, HR (95\% Cl) & - & $0.53(0.40,0.68)^{* * *}$ \\
\hline$\geq 100 \mathrm{~mL}$ decrease in trough $\mathrm{FEV}_{1}, \mathrm{n}(\%)$ & $42(9)$ & $83(18)$ \\
\hline UMEC/VI vs TIO, HR $(95 \% \mathrm{Cl})$ & - & $0.44(0.31,0.64)^{* * *}$ \\
\hline$\geq 4$-unit SGRQ total score increase, $n(\%)$ & $34(7)$ & $5 \mathrm{I}(\mathrm{II})$ \\
\hline UMEC/VI vs TIO, HR $(95 \% \mathrm{Cl})$ & - & $0.64(0.4 \mathrm{I}, 0.99)^{*}$ \\
\hline
\end{tabular}

Notes: $* P<0.05$. $* * * P<0.001$. a Defined as an acute worsening of COPD symptoms requiring the use of additional treatment including oral corticosteroids, antibiotics, emergency department treatment, or hospitalization. ${ }^{b}$ Any first exacerbation was also considered a sustained deterioration as patients were withdrawn by protocol following a first event.

Abbreviations: $\mathrm{Cl}$, confidence interval; CID, clinically important deterioration; FEV , forced expiratory volume in I second; HR, hazard ratio; ITT, intent-to-treat; SGRQ, St George's Respiratory Questionnaire; TIO, tiotropium; UMEC, umeclidinium; VI, vilanterol. 
observed $(P<0.01$; Table S3). Similar results were seen for sustained composite CID with the exception that UMEC/VI did not statistically significantly reduce the risk of a sustained composite CID in the concurrent ICS use subgroup versus UMEC (Table S3).

In Study B, the incidence of a first CID with $\mathrm{TIO}$ (55\%-64\%) and UMEC/VI (40\%-43\%) was similar in all subgroups (Table S4). UMEC/VI treatment statistically significantly reduced the risk of a first composite CID in all subgroups versus TIO $(P<0.01)$ (Table S4). Similar results were seen for sustained composite CID (Table S4).

\section{Safety}

The incidences of on-treatment AEs and serious AEs were similar between all treatment groups in both studies. Safety data from both studies have been previously published. ${ }^{4,6}$

\section{Sensitivity analysis of time to first and sustained CID in Study B}

An analysis excluding $\mathrm{FEV}_{1}$ data from the four of seven visits where no SGRQ data were collected was performed. The incidence of a first composite CID and first deterioration in trough $\mathrm{FEV}_{1}$ was $37 \%(\mathrm{n}=169)$ and $15 \%(\mathrm{n}=67)$ with UMEC/VI and 52\% $(\mathrm{n}=236)$ and 27\% $(\mathrm{n}=122)$ with TIO, respectively. UMEC/VI statistically significantly decreased the risk of a composite CID by 39\% (HR: 0.61 [95\% CI: $0.50,0.75] ; P<0.001)$. For a sustained CID, the incidence of a composite CID and deterioration in trough $\mathrm{FEV}_{1}$ with UMEC/VI was $17 \%(n=75)$ and $6 \%(n=26)$ and with TIO was $27 \%(n=123)$ and $11 \%(n=50)$, respectively. The risk of a sustained composite CID was statistically significantly decreased by 45\% (HR: 0.55 [95\% CI: 0.41, 0.73]; $P<0.001$ ). These trends were similar to the primary analysis.

\section{Discussion}

In COPD management, treatment goals aim to reduce symptoms, improve health status, increase exercise tolerance, prevent exacerbations, and decrease disease progression. ${ }^{1}$ As lung function, health status, and exacerbations all contribute to long-term prognosis, COPD guidelines recommend that these parameters be considered when assessing disease progression and severity. ${ }^{1,12-18}$ The results of this exploratory analysis, using a new assessment of clinical deterioration in COPD, showed that $75 \%$ of placebo-treated patients in Study A and 59\% of TIO-treated patients in Study B reported a composite CID during the 24-week study period. The risk of a first CID was reduced with UMEC/VI treatment compared with TIO, UMEC, VI, or placebo.
Considering the individual components of the composite CID measure, deteriorations in lung function were the most commonly reported individual CID, followed by declines in health status and then moderate-to-severe COPD exacerbations. This may be largely explained by the higher frequency of lung function measurements compared with health status in the original 24-week studies and the recruitment of study populations without a history of frequent exacerbations. In Study A, both dual bronchodilation with UMEC/VI and mono-bronchodilation with UMEC reduced the risk of a first composite CID, as well as the risk of a first clinical deterioration of the individual CID parameters (lung function, health status, and moderate-to-severe exacerbations) compared with placebo. Decreases in risk of a first CID were also observed with VI monotherapy, although this did not include a reduction in the risk of moderate-to-severe exacerbations. Overall, the dual bronchodilator UMEC/VI provided greater protection from a first CID and decreased the risk of deteriorations in trough $\mathrm{FEV}_{1}$ compared with UMEC or VI bronchodilator monotherapy, potentially due to improved airway stability.

In Study B, patients treated with UMEC/VI experienced a statistically significant benefit in preventing first composite CID and a first deterioration in all three individual parameters compared with TIO. This suggests that dual bronchodilation provides greater stability than the recommended first-line mono-bronchodilator treatment in symptomatic patients with COPD. ${ }^{1,6}$ These findings also suggest that dual bronchodilation with UMEC/VI, in addition to producing statistically significant improvements in lung function and PROs compared with $\mathrm{TIO},{ }^{6,8}$ also provides greater protection against future deteriorations.

Deteriorations in lung function and health status could be transient or sustained. In Studies A and B, approximately half of all patients who experienced a first deterioration in lung function and a third of patients with a first deterioration in health status also developed a sustained deterioration. In Study A, as with first CID, UMEC/VI and its bronchodilator mono-components reduced the risk of a sustained composite CID compared with placebo. Overall, as with first CID, the greatest reduction in the risk of a sustained CID was with UMEC/VI dual bronchodilation. UMEC/VI provided a statistically significant reduction in the risk of sustained composite CIDs compared with VI and placebo but not with UMEC. In contrast, Study B showed that UMEC/VI compared with the LAMA TIO provided increased protection from a sustained composite CID and individual sustained deteriorations in trough $\mathrm{FEV}_{1}$ and health status. Overall, these 
results suggest increased protection from sustained CID by UMEC/VI compared with bronchodilator monotherapy.

Currently, it is unclear whether sustained CIDs have greater clinical consequences or prognostic implications than first CIDs on patients with COPD, and the long-term impact of a CID on other aspects of COPD morbidity and mortality requires further investigation. Many COPD exacerbations are not reported to health care professionals, remain untreated, and are associated with worse SGRQ scores. ${ }^{17}$ In the current study, it is possible that deteriorations in $\mathrm{FEV}_{1}$ and SGRQ total score could be unreported exacerbations and may also have a detrimental long-term impact. Furthermore, the threshold at which deteriorations are considered clinically significant requires further investigation. The thresholds used in the current study were based on recognized MCIDs for changes in lung function and health status. ${ }^{11,18-20}$ Alternative cutoff points for deterioration could be explored in future studies to improve understanding of the relationship between CID thresholds and long-term outcomes, and whether this varies with the severity of the population. While we postulate that a CID represents a period of disease worsening, prospective long-term studies are required to expand on these initial post hoc findings and increase understanding of the prognostic weighting of each individual component of CID to the risk of future long-term COPD deteriorations.

COPD deteriorations appeared to be largely independent of baseline airflow limitation, although results for concomitant ICS were inconsistent. Patients with symptomatic COPD with both moderate lung function impairment and low exacerbation risk (GOLD B) or severe-to-very-severe lung function impairment and high exacerbation risk (GOLD D) both reported similar rates of a first CID with placebo in Study A and with TIO in Study B. In Study A, higher rates of a first CID with placebo were seen in patients using concomitant ICS (80\%) compared with those not using ICS (69\%). In Study B, patients treated with TIO monotherapy with concurrent ICS use experienced a lower incidence of a first CID (55\%) compared with those not using ICS (64\%). These contrasting findings mean that we can neither confirm nor exclude the possibility that ICS may contribute to the stabilization of COPD, and further investigation is required to address this question.

Independent of the influence of GOLD group or concurrent ICS use, dual and mono-bronchodilators reduced the risk of first and sustained composite CID compared with placebo in Study A. Additionally, in Study A, UMEC/VI treatment resulted in further risk reductions in first composite CID compared with UMEC and VI and sustained composite CID compared with VI in the GOLD D and concurrent ICS use subgroups. In Study B, UMEC/VI produced a statistically significant reduction in first and sustained composite CID in all subgroups compared with TIO suggesting that the benefits of UMEC/VI compared with TIO are consistent across a range of patient subgroups with differing COPD symptom burden. Reductions in the risk of a CID with UMEC/VI versus monotherapy were more consistent across subgroups in Study B than Study A. This raises the possibility that there may be differences between bronchodilator monotherapies in the ability to prevent a CID.

One study limitation was that results were derived from a post hoc analysis of trials not designed to evaluate CIDs. Also, data were obtained from two short-term 24-week studies, which enrolled only patients with moderate-to-verysevere lung function impairment and moderate-to-very-severe COPD symptoms but a low risk of COPD exacerbations. A longer period may be required to determine the effect of dual- and mono-bronchodilator treatments on exacerbations as a component of the CID end point in patients with a low exacerbation risk, while 24 weeks may be sufficient to assess patients with a higher exacerbation risk. Unsurprisingly, deteriorations in lung function and SGRQ total score were more common than exacerbations in this population of patients with a low exacerbation risk. As lung function was assessed at seven clinic visits and SGRQ total score at only three, a sensitivity analysis was performed to exclude lung function data when there was no concurrent SGRQ total score assessment. This analysis was consistent with the primary analysis, suggesting that results for the composite CID end point were not driven by the overrepresentation of the lung function component. Nevertheless, retrospective and prospective studies are required to further validate the CID end point in different populations of patients receiving different treatment combinations. Additionally, it may be beneficial to investigate the impact of including other PROs that respond to pharmacotherapy, such as the transition dyspnea index..22 Finally, it remains to be determined whether these deteriorations are linked with reduced activity levels, associated with poor COPD prognosis. ${ }^{23-26}$

Overall, these results suggest that long-acting dualbronchodilator therapy may provide greater airway stability, and protection from deteriorations in lung function and health status and the occurrence of exacerbations compared with TIO or VI monotherapies. Interestingly, the combination of the short-acting bronchodilators ipratropium and albuterol, which has the same mechanism of action as LAMA/LABA 
combinations, also reduces daily variation in lung function compared with monotherapy. ${ }^{27}$ A reduction in the daily variation of lung function may lead to more consistent long-term reductions in COPD symptoms. ${ }^{17}$

Current COPD management guidelines recommend that lung function, health status, and exacerbations should all be considered when assessing disease progression and severity, ${ }^{1}$ as all have been shown to contribute to long-term prognosis. ${ }^{12-18}$ By using a threshold at which these deteriorations are considered clinically important, the composite first and sustained CID end points may provide new insights on the effects of bronchodilators in COPD management. ${ }^{11,19}$ It is established that UMEC/VI improves lung function, health status, and symptoms compared with placebo or mono-bronchodilator therapy. ${ }^{4,6}$ The current study highlights a new and important aspect of the benefit of dual therapy with UMEC/VI.

\section{Conclusion}

These results show that patients with COPD experience frequent CIDs involving lung function, health status, and the occurrence of moderate-to-severe exacerbations. Dualbronchodilator therapy with UMEC/VI may reduce the risk of these CIDs compared with long-acting bronchodilator monotherapy or placebo.

\section{Acknowledgments}

The authors wish to thank Alex Lowe, PhD, from Fishawack Indicia Ltd, who provided editorial assistance with developing this manuscript (in the form of writing assistance, including development of the initial draft, assembling tables and figures, collating authors' comments, grammatical editing, and referencing), funded by GSK. This study was funded by GSK (GSK analysis \#202065), and used data from two clinical trials which were also funded by GSK (DB2113373/ NCT01313650 and ZEP117115/NCT01777334).

\section{Author contributions}

All authors contributed toward data analysis, drafting and revising the paper and agree to be accountable for all aspects of the work.

\section{Disclosure}

DS has received sponsorship to attend international meetings, honoraria for lecturing or attending advisory boards, and research grants from various pharmaceutical companies including Almirall, AstraZeneca, Boehringer Ingelheim, Chiesi, Genentech, GSK, Glenmark, Johnson \& Johnson,
Merck, NAPP, Novartis, Pfizer, Skyepharma, Takeda, Teva, Theravance, and Verona. MRM-Y acted as a consultant for Almirall, AstraZeneca, Boehringer Ingelheim, Forest Laboratories, GSK, Novartis, Merck, Ono Pharmaceuticals, and Pfizer. LT is a contingent worker at GSK. AI was an employee of GSK at the time of analysis completion and holds stock/shares in GSK. WF and IN are employees of GSK and hold stocks/shares in GSK. The authors report no other conflicts of interest in this work.

\section{References}

1. GOLD. Global strategy for the diagnosis, management and prevention of chronic obstructive pulmonary disease. 2015. Available from: http:// www.goldcopd.com/. Accessed August 12, 2015.

2. Dransfield MT, Bailey W, Crater G, Emmett A, O'Dell DM, Yawn B. Disease severity and symptoms among patients receiving monotherapy for COPD. Prim Care Respir J. 2011;20(1):46-53.

3. Singh D. New combination bronchodilators for chronic obstructive pulmonary disease: current evidence and future perspectives. Br J Clin Pharmacol. 2015;79(5):695-708.

4. Donohue JF, Maleki-Yazdi MR, Kilbride S, Mehta R, Kalberg C, Church A. Efficacy and safety of once-daily umeclidinium/vilanterol 62.5/25 mcg in COPD. Respir Med. 2013;107(10):1538-1546.

5. Celli B, Crater G, Kilbride S, et al. Once-daily umeclidinium/vilanterol 125/25 mcg in COPD: a randomized, controlled study. Chest. 2014; 145(5):981-991.

6. Maleki-Yazdi MR, Kaelin T, Richard N, Zvarich M, Church A. Efficacy and safety of umeclidinium/vilanterol $62.5 / 25 \mathrm{mcg}$ and tiotropium $18 \mathrm{mcg}$ in chronic obstructive pulmonary disease: results of a 24-week, randomized, controlled trial. Respir Med. 2014;108(12):1752-1760.

7. Oba Y, Sarva ST, Dias S. Efficacy and safety of long-acting betaagonist/long-acting muscarinic antagonist combinations in COPD: a network meta-analysis. Thorax. 2016;71(1):15-25.

8. Decramer M, Anzueto A, Kerwin E, et al. Efficacy and safety of umeclidinium plus vilanterol versus tiotropium, vilanterol, or umeclidinium monotherapies over 24 weeks in patients with chronic obstructive pulmonary disease: results from two multicentre, blinded, randomised controlled trials. Lancet Respir Med. 2014;2(6):472-486.

9. Bateman ED, Ferguson GT, Barnes N, et al. Dual bronchodilation with QVA149 versus single bronchodilator therapy: the SHINE study. Eur Respir J. 2013;42(6):1484-1494.

10. Siler TM, Kerwin E, Sousa AR, Donald A, Ali R, Church A. Efficacy and safety of umeclidinium added to fluticasone furoate/vilanterol in chronic obstructive pulmonary disease: results of two randomized studies. Respir Med. 2015;109(9):1155-1163.

11. Jones PW. St George's Respiratory Questionnaire: MCID. COPD. 2005;2(1):75-79.

12. Donaldson GC, Seemungal TA, Bhowmik A, Wedzicha JA. Relationship between exacerbation frequency and lung function decline in chronic obstructive pulmonary disease. Thorax. 2002;57(10):847-852.

13. Schmidt SA, Johansen MB, Olsen M, et al. The impact of exacerbation frequency on mortality following acute exacerbations of COPD: a registry-based cohort study. BMJ Open. 2014;4(12): e006720.

14. Sin DD, Man SF. Chronic obstructive pulmonary disease as a risk factor for cardiovascular morbidity and mortality. Proc Am Thorac Soc. 2005;2(1):8-11.

15. Sin DD, Wu L, Man SF. The relationship between reduced lung function and cardiovascular mortality: a population-based study and a systematic review of the literature. Chest. 2005;127(6):1952-1959.

16. Tockman MS, Pearson JD, Fleg JL, et al. Rapid decline in FEV1. A new risk factor for coronary heart disease mortality. Am J Respir Crit Care Med. 1995;151(2 Pt 1):390-398. 
17. Jones PW, Lamarca R, Chuecos F, et al. Characterisation and impact of reported and unreported exacerbations: results from ATTAIN. Eur Respir J. 2014;44(5):1156-1165.

18. Wilke S, Jones PW, Mullerova H, et al. One-year change in health status and subsequent outcomes in COPD. Thorax. 2015;70(5):420-425.

19. Donohue JF. Minimal clinically important differences in COPD lung function. COPD. 2005;2(1):111-124.

20. Westwood M, Bourbeau J, Jones PW, Cerulli A, Capkun-Niggli G, Worthy G. Relationship between FEV1 change and patient-reported outcomes in randomised trials of inhaled bronchodilators for stable COPD: a systematic review. Respir Res. 2011;12:40.

21. Celli BR, MacNee W. Standards for the diagnosis and treatment of patients with COPD: a summary of the ATS/ERS position paper. Eur Respir J. 2004;23(6):932-946.

22. Witek TJ Jr, Mahler DA. Minimal important difference of the transition dyspnoea index in a multinational clinical trial. Eur Respir J. 2003; 21(2):267-272.
23. Garcia-Rio F, Rojo B, Casitas R, et al. Prognostic value of the objective measurement of daily physical activity in patients with COPD. Chest. 2012;142(2):338-346.

24. Vaes AW, Garcia-Aymerich J, Marott JL, et al. Changes in physical activity and all-cause mortality in COPD. Eur Respir J. 2014;44(5): 1199-1209.

25. Waschki B, Spruit MA, Watz H, et al. Physical activity monitoring in COPD: compliance and associations with clinical characteristics in a multicenter study. Respir Med. 2012;106(4):522-530.

26. Watz H, Waschki B, Meyer T, Magnussen H. Physical activity in patients with COPD. Eur Respir J. 2009;33(2):262-272.

27. Singh D, Zhu CQ, Sharma S, Church A, Kalberg CJ. Daily variation in lung function in COPD patients with combined albuterol and ipratropium: results from a 4-week, randomized, crossover study. Pulm Pharmacol Ther. 2015;31:85-91. 


\section{Supplementary materials}

Table SI Summary of trough FEV, at day 169 and SGRQ total score at day 168 in Study A (ITT population)

\begin{tabular}{|c|c|c|c|c|}
\hline Endpoint & UMEC/VI (n=4I3) & UMEC $(n=4 \mid 8)$ & VI $(n=42 I)$ & Placebo $(n=280)$ \\
\hline Trough FEV , at day $169, \mathrm{n}$ & 330 & 322 & 317 & 201 \\
\hline LS mean (SE), L & $1.406(0.0126)$ & $\mathrm{I} .354(0.0126)$ & $1.31 \mathrm{I}(0.0127)$ & $1.239(0.0158)$ \\
\hline LS mean change (SE), L & $0.171(0.0126)$ & $0.119(0.0126)$ & $0.076(0.0127)$ & $0.004(0.0158)$ \\
\hline SGRQ total score at day $168, \mathrm{n}$ & 317 & 312 & 304 & 192 \\
\hline LS mean (SE) & $4 I .11(0.749)$ & $41.93(0.753)$ & $41.43(0.760)$ & $46.62(0.950)$ \\
\hline LS mean change (SE) & $-8.07(0.749)$ & $-7.25(0.753)$ & $-7.75(0.760)$ & $-2.56(0.950)$ \\
\hline
\end{tabular}

Abbreviations: FEV , forced expiratory volume in I second; ITT, intent-to-treat; LS, least squares; SE, standard error; SGRQ, St George's Respiratory Questionnaire; TIO, tiotropium; VI, vilanterol; UMEC, umeclidinium; L, liters.

Table S2 Summary of trough FEV, at day 169 and SGRQ total score at day 168 in Study B (ITT population)

\begin{tabular}{lll}
\hline Endpoint & UMEC/VI (n=454) & TIO (n=45I) \\
\hline Trough FEV, at day 169, $\mathbf{n}$ & 400 & 388 \\
LS mean (SE), L & $1.457(0.0114)$ & $1.345(0.0115)$ \\
LS mean change (SE), L & $0.205(0.0114)$ & $0.093(0.0115)$ \\
SGRQ total score at day 168, $\mathbf{n}$ & 388 & 374 \\
LS mean (SE) & $41.35(0.538)$ & $43.45(0.548)$ \\
LS mean change (SE) & $-7.27(0.538)$ & $-5.17(0.548)$ \\
\hline
\end{tabular}

Abbreviations: FEV, forced expiratory volume in I second; ITT, intent-to-treat; LS, least squares; SE, standard error; SGRQ, St George's Respiratory Questionnaire; TIO, tiotropium; VI, vilanterol; UMEC, umeclidinium.

Table S3 Summary of first and sustained composite CID by subgroup in Study A (ITT population)

\begin{tabular}{|c|c|c|c|c|}
\hline Deterioration type/subgroup & UMEC/VI (n=4I3) & UMEC $(n=4 \mid 8)$ & VI $(n=42 I)$ & Placebo $(n=280)$ \\
\hline \multicolumn{5}{|l|}{ First deterioration } \\
\hline GOLD B, n (\%) & $89(48)$ & $76(47)$ & $92(58)$ & $76(72)$ \\
\hline vs placebo, $\mathrm{HR}(95 \% \mathrm{Cl})$ & $0.43(0.32,0.58)^{* * *}$ & $0.4 \mathrm{I}(0.29,0.56)^{* * *}$ & $0.56(0.4 \mathrm{I}, 0.76)^{* * *}$ & - \\
\hline UMEC/VI vs UMEC or VI, HR $(95 \% \mathrm{Cl})$ & - & $1.06(0.78,1.44)$ & $0.76(0.57,1.02)$ & - \\
\hline GOLD D, n (\%) & $93(4 I)$ & $133(52)$ & $144(55)$ & $133(76)$ \\
\hline vs placebo, $\mathrm{HR}(95 \% \mathrm{Cl})$ & $0.32(0.25,0.42)^{* * *}$ & $0.50(0.39,0.64)^{* * *}$ & $0.54(0.42,0.68) * * *$ & - \\
\hline UMEC/VI vs UMEC or VI, HR $(95 \% \mathrm{Cl})$ & - & $0.64(0.49,0.84)^{* *}$ & $0.60(0.46,0.78) * * *$ & - \\
\hline Concurrent ICS use, $\mathbf{n}(\%)$ & $85(40)$ & $116(53)$ & $123(58)$ & $110(80)$ \\
\hline vs placebo, $\mathrm{HR}(95 \% \mathrm{Cl})$ & $0.28(0.21,0.38)^{* * * *}$ & $0.46(0.35,0.59)^{* * *}$ & $0.52(0.40,0.68)^{* * *}$ & - \\
\hline UMEC/VI vs UMEC or VI, HR $(95 \% \mathrm{Cl})$ & - & $0.62(0.47,0.82)^{* * *}$ & $0.54(0.4 \mathrm{I}, 0.7 \mathrm{I})^{* * *}$ & - \\
\hline No concurrent ICS use, $n$ (\%) & $97(48)$ & $94(47)$ & $113(54)$ & $99(69)$ \\
\hline vs placebo, $\mathrm{HR}(95 \% \mathrm{Cl})$ & $0.47(0.36,0.63)^{* * *}$ & $0.47(0.35,0.62)^{* * *}$ & $0.57(0.44,0.75)^{* * *}$ & - \\
\hline UMEC/VI vs UMEC or VI, HR $(95 \% \mathrm{Cl})$ & - & $1.02(0.76,1.35)$ & $0.83(0.63,1.09)$ & - \\
\hline \multicolumn{5}{|l|}{ Sustained deterioration ${ }^{\mathrm{a}}$} \\
\hline GOLD B, n (\%) & $50(27)$ & $34(21)$ & $54(34)$ & $50(47)$ \\
\hline vs placebo, $\mathrm{HR}(95 \% \mathrm{Cl})$ & $0.47(0.31,0.69)^{* * *}$ & $0.34(0.22,0.53)^{* * *}$ & $0.63(0.43,0.92)^{*}$ & - \\
\hline UMEC/VI vs UMEC or VI, HR $(95 \% \mathrm{Cl})$ & - & $1.35(0.87,2.09)$ & $0.74(0.50,1.09)$ & - \\
\hline GOLD D, n (\%) & $50(22)$ & $84(33)$ & $89(34)$ & $87(50)$ \\
\hline vs placebo, HR $(95 \% \mathrm{Cl})$ & $0.33(0.23,0.47)^{* * *}$ & $0.57(0.42,0.77)^{* * *}$ & $0.58(0.43,0.78)^{* * *}$ & - \\
\hline UMEC/VI vs UMEC or VI, HR $(95 \% \mathrm{Cl})$ & - & $0.58(0.4 \mathrm{I}, 0.83)^{* *}$ & $0.57(0.4 \mathrm{I}, 0.8 \mathrm{I})^{* *}$ & - \\
\hline Concurrent ICS use, $n$ (\%) & $47(22)$ & $64(29)$ & $80(38)$ & $74(54)$ \\
\hline vs placebo, $\mathrm{HR}(95 \% \mathrm{Cl})$ & $0.3 \mathrm{I}(0.2 \mathrm{I}, 0.44)^{* * *}$ & $0.44(0.3 \mathrm{I}, 0.6 \mathrm{I})^{* * *}$ & $0.60(0.44,0.82)^{* *}$ & - \\
\hline UMEC/VI vs UMEC or VI, HR $(95 \% \mathrm{Cl})$ & - & $0.70(0.48,1.02)$ & $0.5 \mathrm{I}(0.36,0.74)^{* * *}$ & - \\
\hline No concurrent ICS use, $n$ (\%) & $53(26)$ & $54(27)$ & $63(30)$ & $63(44)$ \\
\hline vs placebo, $\mathrm{HR}(95 \% \mathrm{Cl})$ & $0.49(0.34,0.7 \mathrm{I})^{* * *}$ & $0.51(0.36,0.74)^{* * * *}$ & $0.60(0.42,0.85)^{* *}$ & - \\
\hline UMEC/VI vs UMEC or VI, HR ( $95 \% \mathrm{Cl})$ & - & $0.96(0.66, I .4 I)$ & $0.82(0.57,1.19)$ & - \\
\hline
\end{tabular}

Notes: $* P<0.05 ; * * P<0.01 ; * * * P<0.001$. ${ }^{a}$ Any first exacerbation was also considered a sustained deterioration as patients were withdrawn by protocol following a first event.

Abbreviations: $\mathrm{Cl}$, confidence interval; $\mathrm{CID}$, clinically-important deterioration; GOLD, Global initiative for chronic Obstructive Lung Disease classification; HR, hazard ratio; ICS, inhaled corticosteroid; ITT, intent-to-treat; VI, vilanterol; UMEC, umeclidinium. 
Table S4 Summary of first and sustained composite CID by subgroup in Study B (ITT population)

\begin{tabular}{|c|c|c|}
\hline Deterioration type/subgroup & UMEC/VI $(n=454)$ & TIO $(n=45 I)$ \\
\hline \multicolumn{3}{|l|}{ First deterioration } \\
\hline GOLD B, n (\%) & $77(43)$ & $107(6 \mathrm{I})$ \\
\hline UMEC/VI vs TIO, HR $(95 \% \mathrm{Cl})$ & - & $0.61(0.46,0.82)^{* *}$ \\
\hline GOLD D, n (\%) & III (40) & $159(58)$ \\
\hline UMEC/VI vs TIO, HR $(95 \% \mathrm{Cl})$ & - & $0.55(0.43,0.7 \mathrm{I})^{* * *}$ \\
\hline Concurrent ICS use, n (\%) & $102(4 I)$ & $130(55)$ \\
\hline UMEC/VI vs TIO, HR $(95 \% \mathrm{CI})$ & - & $0.64(0.49,0.83)^{* * *}$ \\
\hline No concurrent ICS use, n (\%) & $86(42)$ & $136(64)$ \\
\hline UMEC/VI vs TIO, HR (95\% Cl) & - & $0.52(0.39,0.58)^{* * *}$ \\
\hline \multicolumn{3}{|l|}{ Sustained deterioration ${ }^{\mathrm{a}}$} \\
\hline GOLD B, n (\%) & $37(2 \mathrm{I})$ & $60(34)$ \\
\hline UMEC/VI vs TIO, HR $(95 \% \mathrm{Cl})$ & - & $0.56(0.37,0.84)^{* *}$ \\
\hline GOLD D, n (\%) & $53(19)$ & $90(33)$ \\
\hline UMEC/VI vs TIO, HR $(95 \% \mathrm{Cl})$ & - & $0.5 \mathrm{I}(0.37,0.72)^{* * *}$ \\
\hline Concurrent ICS use, n (\%) & $49(20)$ & $74(31)$ \\
\hline UMEC/VI vs TIO, HR $(95 \% \mathrm{Cl})$ & - & $0.58(0.40,0.83)^{* *}$ \\
\hline No concurrent ICS use, n (\%) & $4 \mid(20)$ & $76(36)$ \\
\hline UMEC/VI vs TIO, HR (95\% Cl) & - & $0.48(0.32,0.70)^{* * *}$ \\
\hline
\end{tabular}

Notes: ${ }^{* * P}<0.01$; ${ }^{* * *} P<0.001$. any first exacerbation was also considered a sustained deterioration as patients were withdrawn by protocol following a first event.

Abbreviations: $\mathrm{Cl}$, confidence interval; CID, clinically important deterioration; GOLD, Global initiative for chronic Obstructive Lung Disease classification; HR, hazard ratio; ICS, inhaled corticosteroid; ITT, intent-to-treat; TIO, tiotropium; VI, vilanterol; UMEC, umeclidinium.

\section{Publish your work in this journal}

The International Journal of COPD is an international, peer-reviewed journal of therapeutics and pharmacology focusing on concise rapid reporting of clinical studies and reviews in COPD. Special focus is given to the pathophysiological processes underlying the disease, intervention programs, patient focused education, and self management protocols.

\section{Dovepress}

This journal is indexed on PubMed Central, MedLine and CAS. The manuscript management system is completely online and includes a very quick and fair peer-review system, which is all easy to use. Visit $\mathrm{http} / / / \mathrm{www}$.dovepress.com/testimonials.php to read real quotes from published authors. 